\title{
EVOLUCIÓN DEL CONSERVADURISMO DE BALANCE EN ARGENTINA Y CHILE: LA INCIDENCIA DE LAS NIIF ${ }^{1}$
}

\author{
EVOLUTION OF BALANCE SHEET \\ CONSERVATISM IN ARGENTINA AND CHILE: \\ THE IFRS INCIDENCE
}

\author{
Carmen Stella Verón Medina ${ }^{2}$ y Silvina Beatriz Marcolini Tulliani ${ }^{3}$
}

\section{RESUMEN}

La adopción de las Normas Internacionales de Información Financiera (NIIF) por parte de la Unión Europea, Australia y Nueva Zelandia en el año 2005, impulsó el proceso de adopción a nivel mundial y en particular en los países latinoamericanos.

La implementación de las NIIF generó el interrogante sobre si, la calidad de la información financiera emitida bajo este cuerpo normativo se incrementaba con respecto a las normas locales de cada país adoptante. Esta calidad de información puede ser medida a partir de la infravaloración sistemática del patrimonio neto contable respecto del valor de mercado de las empresas. De esta forma se investiga si los criterios de reconocimiento y medición de activos, pasivos y resultados utilizados en la preparación de estados financieros bajo las NIIF, resultan más prudentes o conservadores respecto a los preparados bajo la norma local del país que corresponda.

El objetivo del trabajo es analizar la evolución del conservadurismo de las empresas cotizadas argentinas y chilenas, y en particular si la implementación de la NIIF ha incidido en las prácticas contables conservadoras de las empresas analizadas. Una de las medidas del conservadurismo de balance o incondicional es la relación entre el valor contable y el valor de mercado de la empresa. Esta relación se observa a través del ratio Market to Book (MTB) que, con valores superiores a uno reflejaría la existencia de conservadurismo contable. Para ello realizamos un estudio longitudinal que abarca períodos pre y post NIIF, mediante la comparación del ratio MTB de dichos períodos, a través de un test de comparación de medias (Argentina 2008-2016, Chile 2003-2016). Los datos fueron obtenidos de la base de datos Thomson One.

Los resultados muestran que la implementación de las NIIF en Argentina incrementó el sesgo conservador en la preparación de los estados financieros, mientras que en Chile se redujo.

Palabras claves: Conservadurismo contable - NIIF.

Recepción: 28/08/2018. Aprobación: 23/11/2018.

\section{ABSTRACT}

The adoption of the International Financial Reporting Standards (IFRS) by the European Union, Australia and New Zealand in 2005, promoted a worldwide adoption process, in particular in Latin American countries.

1 Este trabajo forma parte del Proyecto 19/E345: "Los efectos económicos y contables de la aplicación de las NIIF". Universidad Nacional de Rosario, Argentina, período 2014-2017.

2 Facultad de Ciencias Económicas y Estadística, Universidad Nacional de Rosario, cveron@fcecon.unr.edu.ar

3 Facultad de Ciencias Económicas y Estadística, Universidad Nacional de Rosario, smarcoli@fcecon.unr.edu.ar 
The implementation of IFRSs raised the question about the improvement in the quality of the financial information under these standards respected to the local standards of each country that adopts them. The quality of information can be measured on the systematic undervaluation of the net book value in relation to the market value of the company. The research focus on the relationship between financial statement under IFRS are more conservative than financial statements under local GAAP.

This paper analyzes the evolution of conservatism in Argentina and Chile, and also analyzes if the implementation of IFRS has influenced the conservative accounting practices of listed companies in these countries. One of the measures of unconditional conservatism is the relationship between the book value and the market value of the company. This relationship is observed through the Market to Book (MTB) ratio, which with values higher than one would reflect the existence of accounting conservatism. The paper presents a longitudinal study that covers pre and post IFRS periods, by comparing the MTB ratio of these periods through a comparison test of means (Argentina 2008-2016, Chile 2003-2016). The data used for the analysis were from the Thompson One database.

The results show that the implementation of IFRS in Argentina increased the conservative bias in the preparation of the financial statements but in Chile decreased.

Keywords: Accounting Conservatism - IFRS.

\section{INTRODUCCIÓN}

La adopción a nivel mundial de las Normas Internacionales de Información Financiera (NIIF), en particular en los países latinoamericanos, fue impulsada por la adopción por parte de la Unión Europea, Australia y Nueva Zelandia a partir del año 2005. Un proceso similar se inició en Latinoamérica a partir del año 2007 para la implementación de las NIIF, pero en cada país con ritmo disímil. La creación a mediados del 2011 del Grupo Latinoamericano de Emisores de Normas de Información Financiera (GLENIF), evidencia el compromiso de América Latina de adoptar las NIIF como lenguaje contable único en la región y así facilitar el desarrollo de los mercados de valores, las inversiones internacionales y la comparabilidad de la información contable. Argentina y Chile inician su proceso de implementación de las NIIF para las sociedades que realizan oferta pública de sus acciones u obligaciones negociables a partir del año 2007. Dicho proceso concluyó con la obligatoriedad de presentar estados financieros conforme a NIIF a partir del año 2012 para las empresas cotizadas argentinas y a partir del año 2010 para las empresas cotizadas chilenas.

La implementación de las NIIF generó el interrogante sobre la mejora en la calidad de la información financiera emitida bajo este cuerpo normativo, y el planteo sobre si la calidad de la información es sinónimo de calidad de las normas. Una de las formas de medir la calidad de la información financiera es el concepto de conservadurismo de balance, o infravaloración sistemática del patrimonio neto contable respecto al valor de mercado de la empresa. De esta forma se investiga si los criterios de reconocimiento y medición de activos, pasivos y resultados utilizados en la preparación de estados financieros bajo las NIIF, resultan más prudentes o conservadores respecto a los preparados bajo la norma local del país que corresponda.

Las prácticas conservadoras pueden distinguirse entre conservadurismo ex ante o incondicional o de balance y ex post o condicional o de resultados. El conservadurismo ex ante o incondicional se deriva principalmente de las normas contables, al aplicar valores históricos para la medición de los activos, no permitir el reconocimiento de determinados activos, como es el caso del capital intelectual, o las estimaciones que debe realizar la gerencia como las vidas útiles y métodos de amortización para los bienes de uso. El conservadurismo ex post o condicional aparece con la oportunidad en el reconocimiento de las ganancias y pérdidas o del deterioro del valor de los activos, que son el resultado de las decisiones de la gerencia.

La revisión de la literatura evidencia no sólo definiciones diferentes para el concepto de conservadurismo, sino también la utilización de distintos modelos para medirlo, que se han modificado a través del tiempo y que además comparan el grado de conservadurismo entre países, entre períodos y entre cuerpos normativos.

El objetivo del trabajo es analizar la evolución del conservadurismo de las empresas cotizadas argentinas y chilenas, y en particular si la implementación de las NIIF ha incidido en las prácticas contables conservadoras de las empresas analizadas. Una de las medidas del conser- 
vadurismo de balance o incondicional es la relación entre el valor contable y el valor de mercado de la empresa. Esta relación se observa a través del ratio Market to Book (MTB) que, con valores superiores a uno reflejaría la existencia de conservadurismo contable. Para ello realizamos un estudio longitudinal que abarca períodos pre y post NIIF, mediante la comparación del ratio MTB de dichos períodos a través de un test de comparación de medias.

Luego de esta introducción, presentamos la revisión de la literatura sobre las prácticas contables conservadoras de balance en períodos pre y post NIIF y la utilización del ratio MTB para su medición. En el apartado II desarrollamos el estudio empírico y sus resultados, sobre una muestra de empresas argentinas y chilenas cotizadas; y en el apartado III exponemos las conclusiones del estudio.

Revisión de la literatura: Belkaoui (1985) plantea que el principio de conservadurismo sostiene que, ante la existencia de dos o más prácticas contables aceptadas, se muestra preferencia por aquella opción que tenga el menor impacto favorable en el patrimonio neto. Más específicamente, implica la preferencia de los menores valores de los activos y las ganancias, y los mayores valores de las deudas y las pérdidas. De esta forma las prácticas conservadoras afectan tanto a los activos y pasivos, como a los ingresos y gastos, y se exteriorizan a través de prácticas contables conservadoras de valoración de activos y pasivos, o de reconocimiento de ingresos y gastos. En otras palabras, el conservadurismo podrá afectar tanto al estado de situación patrimonial o balance como al estado de resultados o cuenta de ganancias y pérdidas. En adelante consideraremos de forma indistinta a los términos conservadurismo y prácticas contables conservadoras.

De acuerdo con Rueda (2006, 58): “Los criterios de reconocimiento y valoración de los elementos de los estados financieros son prudentes o conservadores en tanto implican la subestimación del resultado económico y el valor del patrimonio neto de la empresa por parte de las cifras contables, de acuerdo con la definición tradicional de "anticipar todas las pérdidas, pero no los beneficios" (Bliss, 1924) o de "registrar preferiblemente los menores valores de activos e ingresos, y los mayores valores de pasivos y gastos". Según Belkaoui $(1985,239)$, "Se trata de mantener una posición prudente a la hora de elaborar la información financiera en condiciones de incertidumbre, a fin de proteger los derechos de los acreedores y los inversores en el capital de la empresa".

La distinción que hace Rueda (2006) entre prudencia o conservadurismo ex ante o incondicional o de balance y ex post o condicional o de resultados, basada en la de Pope y Walker (2003), permite establecer la relación entre ambas. Así el conservadurismo ex ante o incondicional se deriva principalmente de la normativa contable profesional cuando define la aplicación de valores históricos para la medición de los activos o impide el reconocimiento de determinados activos, como es el caso del capital intelectual; y de las decisiones de la gerencia tales como los métodos de amortización elegidos para los bienes de uso. El conservadurismo ex post o condicional aparece con la oportunidad en el reconocimiento de las ganancias y pérdidas o del deterioro del valor de los activos, que son el resultado de las decisiones de la gerencia. En este sentido el autor advierte una relación inversa entre el conservadurismo ex ante y ex post, pues la falta de reconocimiento de un activo o su infravaloración (conservadurismo ex ante) influye en el no reconocimiento de los resultados asociados de los activos no reconocidos, o menores resultados por su infravaloración (conservadursimo $e x$ post). De esta forma a mayor conservadurismo $e x$ ante, menor conservadurismo ex post.

De acuerdo con el objetivo del trabajo, la revisión de la literatura se refiere a conservadurismo de balance. Se presentan en primer término los planteos teóricos y a continuación los estudios empíricos clasificados según correspondan a la etapa previa o posterior de implementación de las NIIF.

El trabajo pionero en este sentido fue el desarrollo teórico de Feltham y Ohlson (1995) que modelizó la relación existente entre el valor de mercado de la empresa y los datos contables relativos a los activos financieros y operativos. Esta distinción de activos surge del trabajo de Modigliani y Miller (1958). Las actividades financieras de la empresa que involucran los activos financieros, se desarrollan en mercados relativamente perfectos que harían plausible considerar la existencia de una "contabilidad perfecta" para dichos activos, según la cual su valor de libros coincidiría con el valor de mercado. Esto no ocurriría con las actividades operativas que involucran a los activos operativos, los cuales no serían comercializados individualmente en mercados perfectos. La determinación del resultado contable operativo consideraría los flujos de caja ajustados mediante la aplicación del principio de devengo. Así, la diferencia entre el valor de libros de la empresa y su valor de mercado, estaría determinada por la existencia de activos operativos, y sería evidencia de la 
existencia de prácticas conservadoras en la valoración de dichos activos. Esta diferencia se denomina "fondo de comercio no reconocido". En el mismo sentido reconocen que el valor de la empresa puede ser descompuesto en el valor de los activos financieros, de los activos operativos y del fondo de comercio no reconocido. Si el fondo de comercio no reconocido es distinto de cero en forma consistente y a largo plazo, estaría evidenciando la existencia de prácticas conservadoras en la valoración de activos que no revierten en el tiempo. De esta forma si la relación entre el valor de mercado de la empresa y su valor en libros o de los fondos propios, conocido como el ratio "market to book" (MTB) es mayor que uno, reflejaría la existencia de conservadurismo contable.

Beaver y Ryan (2000) descomponen la inversa del ratio "market to book", denominado ratio "book to market" (BTM), en dos componentes: los sesgos (bias) y los retardos (lags) en los valores de libros. A través de esta descomposición intentan visualizar las variaciones persistentes (permanentes) y temporales del BTM. También pretenden que dicho ratio tenga la habilidad de predecir el ratio de retorno sobre los fondos propios, para lo cual utilizan el método desarrollado por Ryan (1995).

En la conceptualización de sesgos manifiestan que: a) existen cuando el valor en libros es persistentemente mayor (menor) que el valor de mercado, lo cual hace que el BTM sea persistentemente mayor (menor) que uno; b) demuestran que son el resultado de las mediciones de la "contabilidad conservadora", tales como valorar los inventarios al menor valor entre el coste de adquisición y el valor de mercado o la no activación de los gastos de investigación y desarrollo; c) son una diferencia asintótica entre el valor de libros y el de mercado, la cual es mencionada en el trabajo de Feltham y Ohlson (1995); y d) pueden existir en un contexto de certeza. En lo referente a los retardos expresan que a) son aquellas ganancias o pérdidas inesperadas que son reconocidas contablemente a lo largo del tiempo en lugar de ser reconocidas inmediatamente, generando que el BTM sea temporariamente inferior (superior) que su promedio, pero que a través del tiempo tenderá a él. En ausencia de sesgos, este promedio sería igual a uno; b) demuestran que los retardos no están asociados con mediciones de conservadurismo, pues son el resultado de la aplicación del coste histórico; c) siguiendo el trabajo de Ryan (1995) establecen que existen retardos si las ganancias o pérdidas inesperadas no son totalmente reconocidas en el período que acontecen, pero sí lo son a través de un deter- minado número de períodos siguientes; y d) la existencia de retardos requiere un contexto de incertidumbre. Por su parte, los retardos y los sesgos están sujetos a la relación de conservación de efectivo entendida como la suma del resultado final a lo largo de la vida de la empresa, o de un determinado conjunto de transacciones. Esa situación será independiente de la elección contable realizada para su registración. El trabajo permite inducir que los sesgos se refieren a los métodos de valoración de los activos y pasivos, y los retardos a los criterios de reconocimiento de ingresos y gastos. La existencia de alguno de ellos evidenciaría la aplicación de prácticas contables conservadoras en un sentido amplio.

Zhang (2000) es otra de las investigaciones teóricas que examina cómo las prácticas contables conservadoras afectan la relación entre los datos contables y el valor de la empresa. La existencia de prácticas contables conservadoras son asumidas, pero su motivación no es incluida en el desarrollo del modelo. Su existencia hace que el ratio "Price to book" (PTB) exceda a uno. El trabajo aborda dos cuestiones: a) cómo las influencias conservadoras afectan no solo el ratio PTB sino también el "Price to earnings" y el "Price to capitalized earnings"; y b) cómo combinaciones lineales del valor de libros y resultados capitalizados generan estimaciones insesgadas del valor de mercado de la empresa.

A partir de los planteamientos teóricos de Feltham y Ohlson (1995), Beaver y Ryan (2000) y Zhang (2000) se han realizado distintos estudios empíricos sobre el conservadurismo de balance en dos escenarios distintos, el previo y el posterior a la implementación de las NIIF.

Escenario Pre NIIF. Joos y Lang (1994) realizan el análisis en el contexto europeo y pretenden establecer si existen prácticas contables conservadoras en la valoración de activos y pasivos, en los países de Gran Bretaña, Francia y Alemania, durante el período 1982-1990. Consideran a priori que el sistema contable alemán y francés será más conservador que el británico. Los resultados obtenidos no son consistentes con sus argumentaciones.

Joos (1997) analiza las prácticas contables conservadoras de Alemania, Francia y Gran Bretaña, pero avanza sobre Joos y Lang (1994) al analizar si las diferencias entre países son estadísticamente significativas. Para ello utiliza el modelo EBO, al cual introduce variables dummy para identificar a que país corresponde la observación. Los resultados obtenidos son similares a los del trabajo de 1994, tanto en la infravalora- 
ción de los fondos propios como del resultado contable.

El trabajo empírico Givoly y Hayn (2000) se realizó sobre las empresas estadounidenses que cotizan, para los períodos 1950-1998. Se utilizaron dos muestras y los resultados son consistentes con la tendencia del incremento del conservadurismo contable.

La utilización del ratio MTB en Givoly y Hayn (2000), como una aproximación al conservadurismo contable, genera una reflexión adicional respecto a que si los actuales precios de mercado no representasen una burbuja. De ser cierta esta situación, las empresas estarían sobrevaloradas, y el incremento del MTB no sería generado, únicamente, por la utilización de prácticas contables conservadoras. También identifican otras causas del incremento de este ratio, como consecuencia de la disminución de los fondos propios, tales como: reestructuraciones, utilización del método de la compra en las fusiones o el efecto inflacionario.

García y Mora (2004) realizan un estudio sobre ocho países de la Unión Europea (Reino Unido, Alemania, Francia, Suiza, Holanda, Italia, España y Bélgica), para el período comprendido entre los años 1987-2000. Sus resultados encuentran evidencia empírica de que los países pertenecientes al sistema continental son más conservadores en sus prácticas contables que los países pertenecientes al sistema anglosajón. Además señalan que estas prácticas conservadoras podrían verse reducidas mediante la implementación de una normativa contable común, tal como es el caso de las NIIF.

Escenario Post NIIF. Los estudios realizados en la etapa posterior a la implementación de las NIIF son escasos y varios de ellos se han centrado en la particularidad del año 2005 por ser el año de transición de normas locales a NIIF, en la Unión Europea.

Callao et al. (2007) analizan el ratio book to market de las empresas españolas integrantes del Ibex 35 en el año de transición de las normas locales a las NIIF, encontrando evidencia empírica de que el cambio de normativa incremento el conservadurismo de balance. Los autores consideran que estos resultados pueden ser producto de las características culturales de la tradición contable española. Estos resultados son confirmados por Garrido y Vázquez (2011) sobre una muestra ampliada de las empresas españolas del mercado continuo.

En un sentido opuesto los resultados obtenidos por Horton y Serafeim (2010) muestran que la implementación de las NIIF en las empresas británicas no ha modificado su conservadurismo de balance, debido a que las normas locales no presentan diferencias con respecto a las NIIF. De esta forma, confirman el comportamiento esperado de los países pertenecientes al sistema anglosajón en lo referente a que no presentarían variaciones en sus prácticas contables conservadoras.

Iñiguez et al. (2013) analiza el impacto de la implementación de las NIIF en el conservadurismo de balance de las empresas españolas para el período comprendido entre 2000 y 2009, trabajando con dos ventanas de 5 años correspondientes a la etapa pre y post NIIF. Los resultados obtenidos muestran una reducción del sesgo conservador observado en la etapa pre NIIF con respecto al observado en la etapa post NIIF.

La revisión de los trabajos referidos al conservadurismo de balance muestra una estrecha relación entre las normas contables aplicadas y las características culturales de la tradición contable de cada país (continental o anglosajón). En ambos casos observamos que se refieren a factores exógenos que inciden en este tipo de conservadurismo.

El ratio Market to Book (MTB). La relación entre el precio de mercado o de cotización de la acción de una empresa y su valor patrimonial proporcional es conocido como ratio market to book. El MTB refleja la diferencia entre la estimación que realiza el mercado sobre el valor económico de su capital, a través de las expectativas de los inversores; y el valor del capital medido de acuerdo a los principios o normas contables utilizados para la preparación de los estados financieros. La literatura sobre conservadurismo de balance o incondicional utiliza al MTB como una medida del grado de conservadurismo basada en el mercado, que ante valores superiores a 1 evidenciaría prácticas contables conservadoras.

A partir de la revisión de la literatura presentada consideramos que la implementación de las NIIF y por ende la aplicación del valor razonable en la medición de los activos y pasivos, generaría una aproximación al valor de mercado. De esta forma se produciría una reducción del conservadurismo de balance generada por la aplicación de las normas locales. Las normas contables argentinas y chilenas muestran aproximaciones al valor razonable mediante la aplicación de valores corrientes a algunos elementos activos. En consecuencia formulamos la siguiente hipótesis no direccionada ya que la incidencia de la implementación de las NIIF no puede ser anticipada: 
La implementación de las NIIF incidió en las prácticas contables conservadoras de las empresas cotizadas argentinas y chilenas.

\section{METODOLOGÍA MATERIALES Y MÉTODO}

Muestra. A los fines de contrastar la hipótesis formulada, se obtuvo de la base de datos Thomson One el listado de las empresas cotizadas argentinas y chilenas correspondientes a todos los sectores económicos excepto las correspondientes al sector bancario y de seguros. Esta exclusión se debe a que las empresas de estos sectores al momento de realizar este estudio no han adoptado las NIIF para la elaboración de sus estados contables.

En el estudio se analizaron diez años para las empresas argentinas correspondientes al período 2007-2016; y catorce años para las empresas chilenas correspondientes al período 2003-2016. En la elección de estos períodos se contempló que el período Post NIIF fuera equivalente al período Pre NIIF, así los subperíodos fueron de 5 años para Argentina y 7 años para Chile.

En la Tabla 1 se muestra la distribución anual de las empresas analizadas y que configuran un total de 596 observaciones para Argentina y 1898 observaciones para Chile.

Tabla 1. Distribución anual de las empresas seleccionadas.

\begin{tabular}{|c|c|c|c|}
\hline \multicolumn{4}{|c|}{ ARGENTINA } \\
\hline Año & Frecuencia & $\%$ & Acumulado \\
\hline 2007 & 66 & 11,07 & 11,07 \\
\hline 2008 & 67 & 11,24 & 22,32 \\
\hline 2009 & 65 & 10,91 & 33,22 \\
\hline 2010 & 62 & 10,40 & 43,62 \\
\hline 2011 & 61 & 10,23 & 53,86 \\
\hline 2012 & 61 & 10,23 & 64,09 \\
\hline 2013 & 55 & 9,23 & 73,32 \\
\hline 2014 & 54 & 9,06 & 82,38 \\
\hline 2015 & 53 & 8,89 & 91,28 \\
\hline 2016 & 52 & 8,72 & 100,00 \\
\hline TOTAL & 596 & 100,00 & \\
\hline \multicolumn{4}{|c|}{ CHILE } \\
\hline Año & Frecuencia & $\%$ & Acumulado \\
\hline 2003 & 125 & 6,59 & 6,59 \\
\hline 2004 & 130 & 6,85 & 13,44 \\
\hline 2005 & 137 & 7,22 & 20,65 \\
\hline 2006 & 139 & 7,32 & 27,98 \\
\hline 2007 & 142 & 7,48 & 35,46 \\
\hline 2008 & 140 & 7,38 & 42,83 \\
\hline 2009 & 143 & 7,53 & 50,37 \\
\hline 2010 & 137 & 7,22 & 57,59 \\
\hline 2011 & 135 & 7,11 & 64,70 \\
\hline 2012 & 140 & 7,38 & 72,08 \\
\hline 2013 & 139 & 7,32 & 79,40 \\
\hline 2014 & 136 & 7,17 & 86,56 \\
\hline 2015 & 130 & 6,85 & 93,41 \\
\hline 2016 & 125 & 6,59 & 100,00 \\
\hline TOTAL & 1898 & 100,00 & \\
\hline
\end{tabular}

Fuente: Elaboración propia. 
En la Tabla 2, se presentan los estadísticos descriptivos para el ratio MTB considerando el período completo y los subperíodos pre NIIF y post NIIF. Se observa que la media en todos los períodos analizados es superior a 1 lo cual indica la existencia de conservadurismo de balance, de acuerdo a los trabajos analizados en la revisión de la literatura. Con respecto a las empresas argentinas observamos que la media del ratio MTB es 7,48 con un valor mínimo de $-283,33$ y un valor máximo de 423,36 . Un aspecto a considerar es que la media del período post NIIF $(10,14)$ resulta superior al período pre NIIF $(5,20)$. Por su parte las empresas chilenas presentan una media del ratio MTB de 6,57 con un valor mínimo de $-696,11$ y un valor máximo de 5625 . En el mismo sentido la media del período post NIIF $(5,16)$ resulta inferior al período pre NIIF $(7,95)$ y podría ser un indicador de una disminución del sesgo conservador.

Debido a que la media del ratio MTB no es ajena a la influencia de valores extremos que observamos en las empresas de ambos países, hemos calculado la mediana para todos los períodos estudiados. La mediana es una medida de tendencia central que muestra el número central de una serie de datos ordenados, dejando la misma cantidad de observaciones por encima y por debajo de ella. Dadas estas características de la mediana, se trata de un estimador más robusto de la tendencia central de la muestra, pues no resulta afectado por los valores extremos.

Los valores de la mediana que se presentan en la Tabla 2 mantienen la tendencia observada en las medias de los períodos pre y post NIIF.

Tabla 2. Estadísticos descriptivos del ratio MTB.

\begin{tabular}{cccc}
\cline { 2 - 4 } & \multicolumn{3}{c}{ ARGENTINA } \\
\hline & $\begin{array}{c}\text { Período } \\
\text { Completo }\end{array}$ & $\begin{array}{c}\text { Pre NIIF } \\
\mathbf{2 0 0 7 - 2 0 1 1}\end{array}$ & $\begin{array}{c}\text { Post NIIF } \\
\mathbf{2 0 1 2}-\mathbf{2 0 1 6}\end{array}$ \\
\hline Media & 7,48 & 5,20 & 10,14 \\
\hline Mediana & 1,43 & 1,23 & 1,80 \\
\hline Std.Dev. & 37,93 & 36,19 & 39,76 \\
\hline Min & $-283,33$ & $-283,33$ & $-8,45$ \\
\hline Max & 423,36 & 337,52 & 423,36 \\
\hline $\mathbf{N}$ & 596 & 321 & 275 \\
\hline & & CHILE & Post NIIF \\
\hline Media & Período & Pre NIIF & $\mathbf{2 0 1 0 - 2 0 1 6}$ \\
\hline Mediana & 6,57 & $\mathbf{2 0 0 3 - 2 0 0 9}$ & 5,16 \\
\hline Std.Dev. & 1,30 & 7,95 & 1,24 \\
\hline Min & 133,71 & 1,36 & 38,37 \\
\hline Max & $-696,11$ & 184,54 & $-356,84$ \\
\hline N & 5625 & $-696,11$ & 521,85 \\
\hline
\end{tabular}

Fuente: Elaboración propia.

El incremento de los valores de la media y la mediana observados en el período post NIIF pueden ser interpretados como un incremento del conservadurismo de balance de las empre- sas analizadas. De esta forma debemos aplicar pruebas estadísticas que muestren si las diferencias de los valores promedio del ratio MTB son estadísticamente distintas para los períodos 
pre y post NIIF. Los resultados que se obtengan permitirán contrastar la hipótesis de estudio que hemos enunciado.

En este sentido se pueden aplicar pruebas paramétricas o no paramétricas, dependiendo de si los datos para los períodos analizados si distribuyen o no como una normal. De esta forma se aplicó el test de Kolmogorov-Smirnov cuya hipótesis nula es que los datos se distribuyen como una función normal. En resultados no tabulados la prueba resultó estadísticamente significativa para las observaciones de las empresas argentinas y chilenas, y su interpretación es que el ratio MTB no se distribuye normalmen- te, incumpliendo así uno de los supuestos requeridos para aplicar la prueba paramétrica de la $t$ de Student. De acuerdo a lo expuesto, aplicamos la prueba no paramétrica de Mann-Whitney. Los resultados se muestran en la Tabla 3 y observamos que la prueba resultó significativa al $1 \%$ e interpretamos que existe una diferencia estadísticamente significativa de los rangos promedios del ratio MTB para ambos países. Estos resultados evidencian que en los períodos post NIIF respecto a los periodos pre NIIF, en las empresas argentinas las prácticas conservadoras se incrementaron, mientras que en las empresas chilenas disminuyeron.

Tabla 3. Resultados de la prueba de Mann-Whitnney.

\begin{tabular}{|c|c|c|c|c|}
\hline & \multicolumn{4}{|c|}{ ARGENTINA } \\
\hline & \multirow{2}{*}{ Hipótesis } & Pre NIIF & Post NIIF & \multirow{2}{*}{ P-value } \\
\hline & & $2007-2011$ & 2012-2016 & \\
\hline MTB & $M B_{\text {preNIIF }}>\delta^{\prime}<M T B_{\text {postNIIF }}$ & 83866 & 94039 & $0,0000^{* * * x}$ \\
\hline \multirow[t]{4}{*}{$\mathbf{N}$} & & 321 & 275 & \\
\hline & \multicolumn{4}{|c|}{ CHILE } \\
\hline & \multirow{2}{*}{ Hipótesis } & Pre NIIF & Post NIIF & \multirow{2}{*}{ P-value } \\
\hline & & 2003-2009 & 2010-2016 & \\
\hline МТВ & $M^{\prime} B_{\text {preNIIF }}>0 ́<M T B_{\text {post NIIF }}$ & 943903 & 858247 & $0,0024^{* * * x}$ \\
\hline $\mathbf{N}$ & & 956 & 942 & \\
\hline
\end{tabular}

${ }^{* * *}$ Significativo al $1 \%$

Fuente: Elaboración propia.

\section{Análisis adicionales}

En la muestra de ambos países se consideraron todas las empresas que presentaron información en el período analizado pero no se consideró su permanencia durante dicho período. Esta situación puede inducirnos a resultados que estarían incididos por empresas que tuvieron prácticas contables conservadoras en alguno de los años analizados, pero no en todo el período. A los fines de obtener resultados más robustos procedimos a construir una muestra de empresas que tuvieran observaciones para todo el período (Argentina 2007-2016 y Chile 2003-2016). De esta forma la muestra se redujo a 47 empresas argentinas y a 76 empresas chilenas que mostraron permanencia en el período estudiado. En la Tabla 4 , se presentan los descriptivos de esta muestra y observamos que la media y la mediana de las empresas argentinas se incrementó mientras que en las empresas chilenas la media disminuyó y la mediana no presentó variación con respecto a la muestra inicial. En lo referente a los valores extremos se observa que en las empresas argentinas se redujo el valor mínimo, pero se mantuvo el valor máximo, mientras que en las empresas chilenas disminuyeron ambos extremos. Finalmente, las medidas de dispersión muestran un incremento en las empresas argentinas y una disminución en las empresas chilenas, presentando una coherencia con las variaciones de los valores mínimo y máximo observados. 
Tabla 4. Estadísticos descriptivos de las empresas con permanencia en el perído analizado.

\begin{tabular}{ccc}
\cline { 2 - 3 } & $\begin{array}{c}\text { ARGENTINA } \\
\mathbf{2 0 0 7 - 2 0 1 6}\end{array}$ & $\begin{array}{c}\text { CHILE } \\
\mathbf{2 0 0 3 - 2 0 1 6}\end{array}$ \\
\hline Media & 8,55 & 3,03 \\
\hline Mediana & 1,47 & 1,30 \\
\hline Std.Dev. & 39,43 & 14,44 \\
\hline Min & $-87,91$ & $-4,95$ \\
\hline Max & 423,36 & 314,04 \\
\hline $\mathbf{N}$ & 470 & 1064 \\
\hline
\end{tabular}

Fuente: Elaboración propia.

Los resultados de la prueba no paramétrica de Mann-Whitney se presentan en la Tabla 5 y resultó significativa al 1\%, mostrando que existe una diferencia estadísticamente significativa de los rangos promedios del ratio MTB en los períodos pre y post NIIF. Se confirmó el sentido de los rangos promedios para las empresas argentinas y chilenas, pues para las primeras muestra un incremento en las prácticas conservadoras, mientras que para las segundas muestra una disminución.

Tabla 5. Prueba de Mann-Whitnney en las empresas con permanencia en el período analizado.

\begin{tabular}{|c|c|c|c|c|}
\hline & \multicolumn{4}{|c|}{ ARGENTINA } \\
\hline & \multirow{2}{*}{ Hipótesis } & Pre NIIF & Post NIIF & \multirow{2}{*}{ P-value } \\
\hline & & 2007-2011 & 2012-2016 & \\
\hline МТВ & $M T B_{\text {preNIIF }}>0 ́<M T B_{\text {postNIIF }}$ & 47293 & 63391 & $0,0000^{* x+1}$ \\
\hline \multirow[t]{4}{*}{$\mathbf{N}$} & & 235 & 235 & \\
\hline & \multicolumn{4}{|c|}{ CHILE } \\
\hline & \multirow{2}{*}{ Hipótesis } & Pre NIIF & Post NIIF & \multirow{2}{*}{ P-value } \\
\hline & & 2003-2009 & 2010-2016 & \\
\hline МТВ & $M T B_{\text {preNIIF }}>0<<M T B_{\text {postNIIF }}$ & 306307 & 260273 & $0,0000^{*+1+}$ \\
\hline $\mathbf{N}$ & & 532 & 532 & \\
\hline
\end{tabular}

${ }^{* * *}$ Significativo al $1 \%$

Fuente: Elaboración propia.

\section{CONCLUSIONES, DISCUSIÓN}

Los resultados obtenidos en los distintos análisis nos permiten afirmar que la implementación de las NIIF incidió en las prácticas conservadoras de balance de las empresas argentinas y chilenas. En las empresas argentinas se produjo un incremento del conservadurismo de balance, mientras que en las empresas chilenas se observó una disminución del conservadurismo de balance.

Estos resultados nos permiten inferir que la aplicación de las NIIF por parte de las empresas chilenas y su correspondiente valor razonable, generó un incremento del patrimonio neto en el período post NIIF, reduciendo los valores 
del ratio MTB. En el mismo sentido entendemos que las normas locales chilenas priorizaban la aplicación del criterio de medición al costo en el período pre NIIF.

En el caso de las empresas argentinas el hecho de que las normas locales presentaran aproximaciones a mediciones a valores corrientes, no resultó relevante al momento de implementar las NIIF. Sirva como ejemplo la aplicación de la NIC 2 que obliga a medir al costo a los Inventarios (bienes de cambio). En el mismo sentido la aplicación de la NIC 38 no permitió el reconocimiento como Activos Intangibles a los gastos de investigación y desarrollo. Estas circunstancias implican una reducción del patrimonio neto con respecto al período pre NIIF, impactando directamente en el ratio MTB.

En la revisión de la literatura observamos que los resultados sobre el conservadurismo de balance en los períodos previos y posteriores a la implementación de las NIIF, no son concluyentes (Callo et al., 2007; Garrido y Vázquez, 2011; Iñiguez et al. 2013 y Horton y Serafeim, 2010). En este sentido nuestros resultados con respecto a las empresas argentinas son similares a los obtenidos por Callao et al. (2007) y Garrido y Vázquez (2011) en estudios realizados sobre empresas españolas, que muestran que la implementación de las NIIF incrementó el conservadurismo de balance en el contexto español. Por su parte nuestros resultados con respecto a las empresas chilenas son similares a los de Iñiguez et al. (2013) pues muestra una reducción del sesgo conservador en el período post NIIF.

\section{REFERENCIAS BIBLIOGRÁFICAS}

ARCE, M. \& MORA, A. (2002). “Empirical evidence of the effect of European accounting differences on the stock market valuation of earnings and book value", European Accounting Review, 11, 1-27.

BASU, S. (1997). "The conservatism principle and the asymmetric timeliness of earnings", Journal of Accounting and Economics, 24, 3-37.

BEAVER, W.H. \& RYAN, S.G. (2000). "Biases and lags in book value and their effects on the ability of the book-to-market ratio to predict book return on equity", Journal of Accounting Research, $38,127-148$.

BELKAOUI, A. (1993). Accounting theory, Tercera edición, Harcourt Brace Jovanovich, Orlando, Florida.

CALLAO, S., JARNE, J. \& LAINEZ, J. (2007). "Adoption IFRS in Spain. Effect pm the comparability and relevance of financial reporting", Journal of International Accounting, Auditing and Taxation, 16(2) 148-178.

FELTHAM, G.A. \& OHLSON, J.A. (1995). “Valuation and clean surplus accounting for operating and financial activities", Contemporary Accounting Research, 11, 689-731.

GARCIA, J.M. \& MORA, A. (2002). "Balance Sheet vs. Earningsconservatism in Europe", XXV Congreso de la Asociación Europea de Contabilidad, Copenhagen.

GARCIA, J.M. \& MORA, A. (2004). "Balance Sheet vs. Earnings conservatism in Europe", European Accounting Review, 13(2), 261-292.

GARCIA, J.M., RUEDA, J.A. \& VAZQUEZ, P. (2008). "Conservadurismo del resultado contable bajo las Normas Internacionales de Contabilidad. Un estudio comparativo", Revista Española de Financiación y Contabilidad, XXXVII(138), 197210.

GARCIA, J.M. \& MORA, A. (2003). “La incorporación asimétrica de noticias al resultado contable en un contexto europeo: evidencia empírica", Revista Española de Financiación y Contabilidad, XXXII(116), 235-264.

GARCIA J.M., GARCIA, B. y PEÑALVA, F. (2014). "Information consequences of accounting conservatism". European Accounting Review, 23(2), 173-198.

GARCÍA, J.M. (2002)."Conservadurismo contable en Europa", Tesis Doctoral, UV.

GARCIA-OSMA, B. E POPE, P. (2011). Strategic balance sheet adjustments under first.time IFRS adoption and the consequences for earnings quality, Working Paper, January, Available at http://dx. doi.org/10.2139/ssrn.1735009

GARRIDO, P \& VAZQUEZ, P. (2011). “The transition to international financial reporting standards in Spain: relevance and timeliness of adjustments", International Journal of Accounting, Auditing and Performance Evaluation, 7(4), 287302. 
GIGLER, F.B. \& HEMMER, T. (2001). “Conservatism, optimal disclosure policy, and the timeliness of financial reports", The Accounting Review, 76, 471-493.

GINER, B. (2001); “La utilidad de la información contable desde la perspectiva del mercado: ¿evolución o revolución en la investigación?", Revista de Contabilidad, 4(7), 21-52.

GIVOLY, D. \& HAYN, C. (2000). “The changing time-series properties of earnings, cash flows and accruals: Has financial reporting become more conservative?", Journal of Accounting and Economics, 29, 287-320.

HJELSTRÖM, A. E SCHUSTER, W. (2011). “Standards, management incentives and accounting practice-lessons from the IFRS transition in Sweden", Accounting in Europe, 8(1), 69-88.

HORTON, J. \& SERAFEIM, G. (2010). “Market reaction to and valuation of IFRS reconciliation adjustments: first evidence from the UK"; Review of Accounting Studies, 15(4); 725-751.

INTERNATIONAL ACCOUNTING STANDARDS BOARD, IASB (1999). "Marco conceptual para la preparación y presentación de los estados financieros", Instituto Mexicano de Contadores Públicos, México.

IÑIGUEZ, R., POVEDA, F. \& VAZQUEZ, P. (2013). “Evolución del conservadurismo de balance con la implantación de las Normas Internacionales de Información Financiera: aplicación al caso de España", Revista Española de Financiación y Contabilidad, XLII(160) 453-486.

JOOS, P.R. (1997). The stock market valuation of earnings and book value across international accounting systems, Tesis Doctoral, UMI.

JOOS, P.R. \& LANG, M. (1994). “The effects of accounting diversity: evidence from the European Union", Journal of Accounting Research, 32, 141-168.

MODIGLIANI, F., \& MILLER, M. (1958). “The Cost of Capital, Corporation Finance and the Theory of Investment", The American Economic Review, 48(3), 261-297.

OHLSON, J.A. (1995). "Earnings, book values, and dividends in equity valuation", Contemporary Accounting Research, 11, 661-687.
POPE, P.F. \& WALKER, M. (1999). “International differences in the timeliness, conservatism and classification of earnings", Journal of Accounting Research, 37, supplement, 53-87.

POPE, P.F. \& WALKER, M. (2003). "Ex-ante and ex-post accounting conservatism, asset recognition and asymmetric earnings timeliness", Working paper, Lancaster University and University of Manchester.

RUEDA, J. (2006). “Prudencia del resultado bajo normas internacionales de contabilidad: un estudio comparado en Europa", Cuadernos de Estudios Empresariales, 16, 55-79.

RYAN, S. (1995). "Model of Accrual Measurement with Implications for the Evolution of the Book-to-Market Ratio", Journal of Accounting Research, Spring, 96-112.

WATTS, R. L. (2002). "Conservatism in Accounting Part I: explanations and implications", Accounting Horizons, 17(3), 207-221.

WATTS, R. L. (2002). “Conservatism in Accounting Part II: evidence and research opportunities", Accounting Horizons, 17(4), 287-301.

WATTS, R.L. \& ZIMMERMAN, J.L. (1978). “Towards a positive theory of the determination of accounting standards", Accounting Review, 53 (1), 112-134.

WATTS, R.L. \& ZIMMERMAN, J.L. (1986). Positive Accounting Theory, Prentice Hall International, London.

ZHANG, X. (2000). “Conservative accounting and equity valuation", Journal of Accounting and Economics, 29, 125-149. 